\title{
Case Report \\ Effect of Pravastatin as an Adjunctive Therapeutic for Mitral Insufficiency with Hyperlipidemia in a Dog
}

\author{
Yu Sahashi, ${ }^{1,2}$ Miwako Sahashi, ${ }^{1}$ and Yoshiaki Hikasa $\mathbb{D}^{2}$ \\ ${ }^{1}$ Sahashi Veterinary Hospital, 23-10 Kitabatake, Ueno, Inagawa, Kawabe-gun, Hyogo 666-0244, Japan \\ ${ }^{2}$ Laboratory of Veterinary Internal Medicine, Department of Clinical Veterinary Science, Joint Graduate School of \\ Veterinary Sciences, Tottori University, 4-101 Koyama Minami, Tottori 680-8553, Japan \\ Correspondence should be addressed to Yoshiaki Hikasa; hikasa@tottori-u.ac.jp
}

Received 19 May 2021; Accepted 23 August 2021; Published 7 September 2021

Academic Editor: Nektarios D. Giadinis

Copyright (c) $2021 \mathrm{Yu}$ Sahashi et al. This is an open access article distributed under the Creative Commons Attribution License, which permits unrestricted use, distribution, and reproduction in any medium, provided the original work is properly cited.

Pravastatin (PS) has been found to increase left ventricle (LV) expansion capacity and decrease LV constriction and left atrial pressure in healthy dogs. To date, there are no available reports on the effects of PS in dogs with hypercholesterolemia with chronic heart failure (CHF). This case report demonstrates a successful long-term treatment plan using PS in a dog suffering from mitral insufficiency with hyperlipidemia. A 12-year-old, castrated male Chihuahua dog had mitral insufficiency with hyperlipidemia. The dog presented with symptoms of chronic coughing. PS was orally administered (1 mg/kg, SID) in addition to general treatment for mitral insufficiency. The follow-up period was 375 days. PS administration decreased the heart rate (HR), vertebral heart size (VHS), and N-terminal probrain natriuretic peptide (NT-proBNP) concentration of the dog. In addition, PS administration also improved chronic cardiac failure induced by mitral insufficiency and hyperlipidemia. This report suggests that PS can be useful as an adjunctive therapeutic for dogs with hypercholesterolemia with mitral insufficiency.

\section{Introduction}

Statins are 3-hydroxy-3-methyl-glutaryl-CoA (HMG-CoA) reductase inhibitors that reduce blood cholesterol levels by inhibiting the HMG-CoA reductase in the mevalonic acid pathway [1]. In addition, this group of medications has been shown to have multifaceted effects, including antimyocardial hypertrophy, anti-inflammatory effects, and antioxidant activity [1]. Statins activate the P13 kinaseAkt pathway by inhibiting the mevalonate pathway. Statins also activate endothelial nitric oxide synthase (eNOS), followed by an increase in nitric oxide (NO) production $[1,2]$. Pravastatin (PS), a statin medication, has been reported to prevent cardiomyocyte cell death via an increase in NO release, a decrease in myocyte endothelin1 production, and an increase in protein kinase Akt activation [3]. Moreover, statins have been described to modulate both the neurohormonal activation and the autonomic nervous system by activating eNOS and increasing NO production [1]. For example, it has been shown that elevated cholesterol levels are associated with the overexpression of angiotensin II type 1 receptors [4] and that statins decrease the levels of these receptors, thus resulting in both decreased angiotensin II-mediated vasoconstriction and enhanced response to angiotensin receptor blockers [5-7]. Furthermore, simvastatin has been reported to normalize sympathetic outflow and reflex regulation in rabbits with chronic heart failure (CHF) [8]. Therefore, these drugs may be useful for the treatment of CHF with hypercholesterolemia in dogs. In a previous study, we have reported that PS increases left ventricle (LV) expansion capacity and decreases LV constriction and left atrial pressure in healthy dogs [9]. Consequently, we suggested that PS may be effective in improving heart failure with LV diastolic dysfunction or elevated left atrial pressure in dogs. To date, no reports are available on the effects of PS in dogs with hypercholesterolemia with CHF. This case report demonstrates a successful long-term treatment plan using PS in a dog suffering from mitral insufficiency with hyperlipidemia. 
TABLE 1: Hematological, serum biochemical, and cardiac biomarker parameters after pravastatin administration.

\begin{tabular}{|c|c|c|c|c|c|c|c|c|c|c|c|c|}
\hline \multirow{2}{*}{ Variables } & \multirow{2}{*}{$\begin{array}{l}\text { Reference } \\
\text { Range }\end{array}$} & \multicolumn{11}{|c|}{ Time (days) } \\
\hline & & 0 & 11 & 30 & 45 & 65 & 96 & 121 & 150 & 211 & 283 & 375 \\
\hline White blood cells $\left(\times 10^{3} / \mathrm{mm}^{3}\right)$ & $6-17$ & 12.0 & - & 10.1 & - & 11.1 & 10.7 & 8.7 & 9.1 & 10.6 & 9.4 & 11.2 \\
\hline Red blood cells $\left(\times 10^{4} / \mathrm{mm}^{3}\right)$ & $550-850$ & 652 & - & 656 & - & 723 & 758 & 645 & 718 & 676 & 695 & 686 \\
\hline Packed cell volume (\%) & $37-55$ & 45.1 & - & 45.1 & - & 49.9 & 51.6 & 44.3 & 49.9 & 46.4 & 47.3 & 47.0 \\
\hline Alanine aminotransferase (U/L) & $10-100$ & 59 & - & 102 & - & 109 & 112 & 118 & 123 & 105 & 92 & 98 \\
\hline Aspartate aminotransferase (U/L) & $0-50$ & 22 & - & 25 & - & 27 & 28 & 24 & 30 & 29 & 28 & 30 \\
\hline Alkaline phosphatase (U/L) & $23-212$ & 443 & - & 297 & - & 319 & 361 & 330 & 367 & 303 & 244 & 242 \\
\hline Blood urea nitrogen $(\mathrm{mg} / \mathrm{dL})$ & $7-27$ & 14.3 & - & 16.3 & - & 17.2 & 13.4 & 17.7 & 19.9 & 17.2 & 14.4 & 18.2 \\
\hline Creatinine $(\mathrm{mg} / \mathrm{dL})$ & $0.5-1.8$ & 0.6 & - & 0.8 & - & 0.8 & 0.8 & 0.6 & 0.6 & 0.7 & 0.8 & 0.7 \\
\hline Triglyceride (mg/dL) & $17-79$ & 577 & 396 & 180 & 178 & 424 & 402 & 209 & 205 & 263 & 162 & 294 \\
\hline Total cholesterol (mg/dL) & $111-320$ & 360 & 281 & 274 & 273 & 279 & 295 & 295 & 317 & 256 & 253 & 239 \\
\hline Creatine kinase $(\mathrm{U} / \mathrm{L})$ & $10-200$ & 60 & 75 & 69 & 75 & 98 & 75 & 63 & 70 & 79 & 75 & 166 \\
\hline Serum N-terminal probrain natriuretic peptide (pmol/L) & $<900$ & 2449 & 1352 & 1117 & 1402 & 1305 & 1209 & 1020 & 1058 & 988 & 2704 & 2146 \\
\hline
\end{tabular}

\section{Case Presentation}

A 12-year-old, castrated male Chihuahua dog weighing $4.2 \mathrm{~kg}$ was referred to the hospital with a diagnosis of chronic coughing. The medical history of the dog included tracheal collapse. Physical examinations revealed a grade III murmur and cough. Respiration rate was 20 breaths/min. The dog was diagnosed with hyperlipidemia and mitral insufficiency whose severity was classified as II b by the International Small Animal Cardiac Health Council. Consequently, the administration of alacepril $(1.5 \mathrm{mg} / \mathrm{kg}$, SID) and a low-fat meal were initially prescribed. The subsequent treatment was continued for a period of two months. However, cardiomegaly continued to progress, whereas the frequency of coughing was not reduced. Moreover, hyperlipidemia was not improved. Abdominal ultrasonography revealed biliary debris that was gradually increasing in size. Consequently, PS $(1 \mathrm{mg} / \mathrm{kg}$, SID) was prescribed. Following PS administration, the clinical signs were evaluated, and hematologic, serum biochemical, and radiologic examinations and echocardiographic and circulation parameter measurements were performed.

Cough disappeared one week after PS administration. In addition, blood biochemical tests revealed that the total cholesterol, triglyceride, and N-terminal probrain natriuretic peptide (NT-proBNP) concentrations were significantly decreased from Day 11 to Day 211 (Table 1). An increase in creatine kinase value was not observed throughout the administration period. Additional hematologic and biochemical findings indicating renal or hepatic failures and adverse effects were not observed up to 375 days after PS administration (Table 1).

Furthermore, the radiographic vertebral heart size (VHS) decreased after PS administration, as shown in Table 2. With respect to the echocardiographic and circulation variables, decreases in heart rate (HR) and left atrium/aorta ratio (LA/Ao) were observed during PS administration, whereas LV fractional shortening (FS) and LV ejection fraction (EF) increased from Day 30 to Day 150 after PS administration (Table 2). No marked changes in the peak velocity of early dia- stolic transmitral flow $(E)$, peak velocity of late transmitral flow $(A), E / A$ ratio, and deceleration time of early diastolic transmitral flow $\left(\mathrm{DT}_{\mathrm{E}}\right.$ ) were observed during PS administration. Stroke volume (SV) and cardiac output (CO) decreased on Days 30 and 45 after PS administration but thereafter increased and maintained well. No substantial changes in the maximum systolic mitral regurgitation velocity (MRmax) were identified after PS administration. The maximum systolic tricuspid regurgitation velocity (TRmax) and systolic pulmonary arterial pressure (sPA), calculated using the modified Bernoulli equation $\left(4 \mathrm{x} \mathrm{TRmax}^{2}+10\right)$, both decreased after PS administration (Table 2 ). The peak velocity of early diastolic mitral annular motion (Em)/peak velocity of diastolic mitral annular motion (Am) ratio increased during PS administration compared with preadministration. E/Em decreased on Days 34 and 65 after PS administration but increased after Day 96, and a higher value was then observed (Table 2).

\section{Discussion}

In dogs with mitral insufficiency, increased HR, LA/Ao ratio and serum NT-proBNP concentration, and pulmonary hypertension $(\mathrm{PH})$ can be considered negative prognostic factors $[10,11]$. However, our case study reveals that $\mathrm{HR}$ apparently decreased after PS administration. This may be due to the PS-induced inhibition of the sympathetic nerve activity via blocking angiotensin II-mediated activity [1]. In addition, VHS, LA/Ao ratio, sPA, and serum NT-proBNP concentration decreased after PS administration, which could be improved with the prognosis.

Furthermore, we identified that both SV and $\mathrm{CO}$ decreased on Days 30 and 45 after PS administration but thereafter increased. In fact, SV reached a higher value compared with before PS administration. As statins activate eNOS followed by an increase in NO production and inhibit the Rho GTPases resulting in peripheral arterial vasodilatation $[1,2]$, we speculated that the increase in $\mathrm{CO}$ may be due to the improved heart failure facilitated by cardiac afterload reduction after PS administration. In this case, however, CO did not apparently increase after PS administration, 


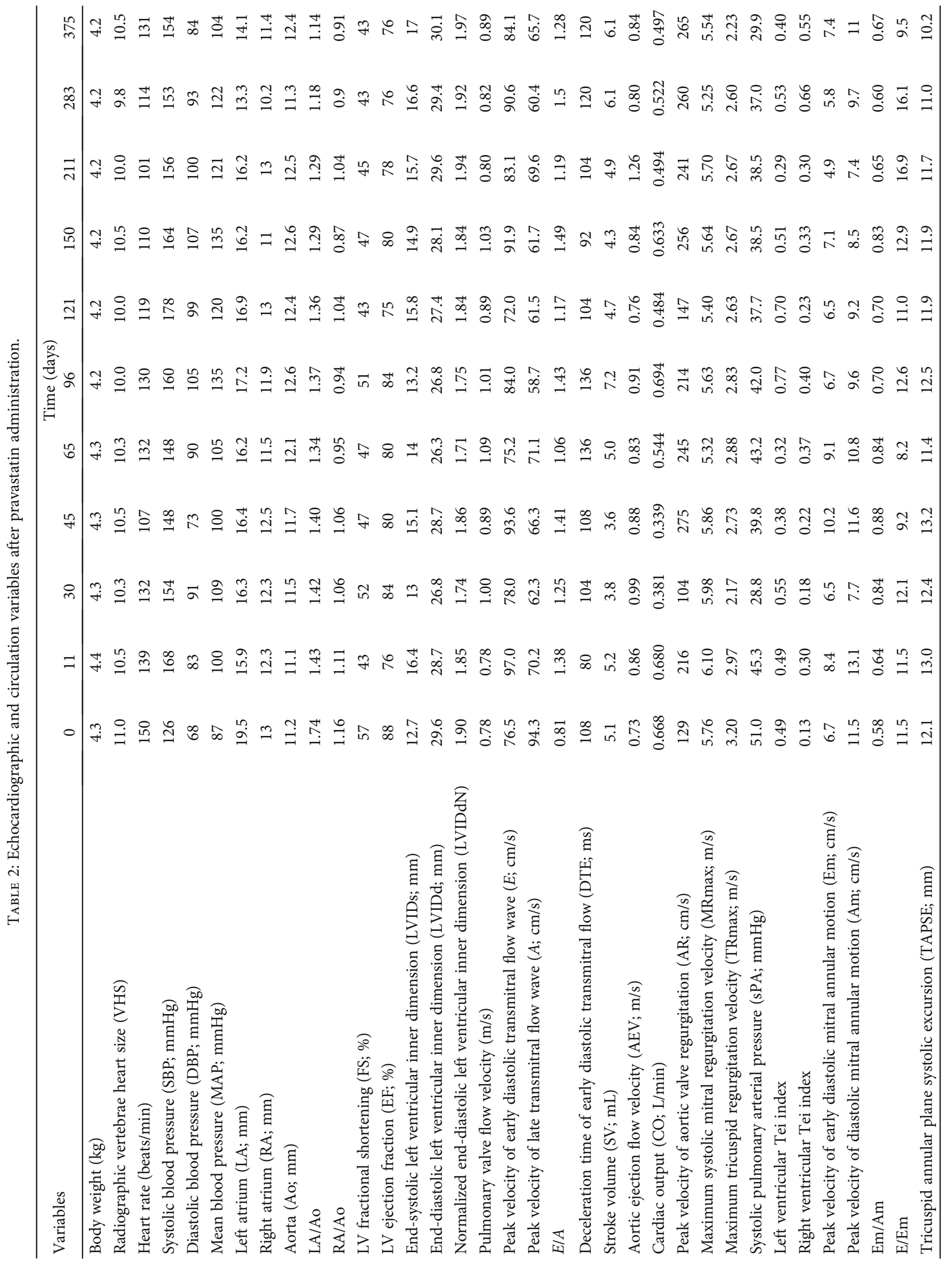


which could be attributed to the decrease in HR after PS administration.

Furthermore, MRmax did not markedly change from the baseline value. In contrast, both TRmax and sPA decreased after PS administration, which may be caused by the pulmonary artery dilation from the inhibitory effect of statins on Rho kinase [12]. In addition, because the decreased eNOS expression [12] and increased endothelin-1 expression [13] have been identified as causes of PH, both PS-induced eNOS activation [2] and decreased ET-1 expression [3] may be involved in sPA reduction. E/Em temporarily decreased after PS administration, thus suggesting that PS administration decreases left atrial pressure reduction. Finally, the decrease in serum NT-proBNP observed after the administration of PS may be associated with the reduction of cardiac load facilitated by the stain-induced inhibition of angiotensin II type 1 receptor expression and vascular contraction by angiotensin II [1].

In this case, coughing was apparently improved after PS administration. This may be due to cardiac load reduction as evidenced by the decrease in VHS and LA/Ao ratio after PS administration. In contrast, statins are known to have antiinflammatory effects by acting on several pathways or factors, such as cholesterol biosynthesis pathway, Ras and Rho kinase, nuclear factor- $\kappa \mathrm{B}$ and activator protein-1-mediated proinflammatory pathways, and peroxisome proliferator-activated receptor and Kruppel-like factor-2 [14]. Therefore, coughing improvement following PS administration may be due to the multifaceted effects of statins, including cardiac load reduction and anti-inflammatory effects.

In conclusion, PS administration in addition to the general treatment for mitral insufficiency with hyperlipidemia in a dog was found to decrease tachycardia and left atrial pressure and also improved hyperlipidemia and $\mathrm{PH}$ without reducing blood pressure. Moreover, the clinical symptoms of chronic cardiac failure were also improved. Therefore, PS administration may be effective as an adjunctive therapy in improving cardiac function and lipid metabolism in dogs with both mitral insufficiency and hyperlipidemia.

\begin{tabular}{|c|c|}
\hline \multicolumn{2}{|c|}{ Abbreviations } \\
\hline A: & Peak velocity of late transmitral flow \\
\hline Ao: & Aorta \\
\hline Am: & $\begin{array}{l}\text { Peak velocity of diastolic mitral } \\
\text { annular motion }\end{array}$ \\
\hline CHF: & Chronic heart failure \\
\hline CO: & Cardiac output \\
\hline $\mathrm{DT}_{\mathrm{E}}:$ & $\begin{array}{l}\text { Deceleration time of early diastolic } \\
\text { transmitral flow }\end{array}$ \\
\hline E: & $\begin{array}{l}\text { Peak velocity of early diastolic } \\
\text { transmitral flow }\end{array}$ \\
\hline EF: & Ejection fraction \\
\hline Em: & $\begin{array}{l}\text { Peak velocity of early diastolic mitral } \\
\text { annular motion }\end{array}$ \\
\hline eNOS: & Endothelial nitric oxide synthase \\
\hline & Fractional shortening \\
\hline HMG-CoA: & 3-Hydroxy-3-methyl-glutaryl-CoA \\
\hline HR: & Heart rate \\
\hline
\end{tabular}

$\begin{array}{ll}\text { LA: } & \text { Left atrium } \\ \text { LV: } & \text { Left ventricle } \\ \text { MRmax: } & \text { Maximum systolic mitral } \\ & \text { regurgitation velocity } \\ \text { NO: } & \text { Nitric oxide } \\ \text { NT-proBNP: } & \text { N-terminal probrain natriuretic peptide } \\ \text { PH: } & \text { Pulmonary hypertension } \\ \text { PS: } & \text { Pravastatin } \\ \text { sPA: } & \text { Systolic pulmonary arterial pressure } \\ \text { SV: } & \text { Stroke volume } \\ \text { TRmax: } & \text { Maximum systolic tricuspid } \\ & \text { regurgitation velocity } \\ \text { VHS: } & \text { Vertebral heart size. }\end{array}$

\section{Data Availability}

The clinical data used to support the findings of this case are included within the text and in tables.

\section{Conflicts of Interest}

The authors declare that they have no conflicts of interest.

\section{Acknowledgments}

The authors thank the dog's owner for involvement in the study.

\section{References}

[1] K. Ramasubbu, J. Estep, D. L. White, A. Deswal, and D. L. Mann, "Experimental and clinical basis for the use of statins in patients with ischemic and nonischemic cardiomyopathy," Journal of the American College of Cardiology, vol. 51, no. 4, pp. 415-426, 2008.

[2] Y. Rikitake and J. K. Liao, "Rho GTPases, statins, and nitric oxide," Circulation Research, vol. 97, no. 12, pp. 1232-1235, 2005.

[3] S. Verma, V. Rao, R. D. Weisel et al., "Novel cardioprotective effects of pravastatin in human ventricular cardiomyocytes subjected to hypoxia and reoxygenation: beneficial effects of statins independent of endothelial cell," Journal of Surgical Research, vol. 119, no. 1, pp. 66-71, 2004.

[4] K. Strehlow, S. Wassmann, M. Böhm, and G. Nickenig, "Angiotensin AT1 receptor over-expression in hypercholesterolaemia," Annals of Medicine, vol. 32, no. 6, pp. 386-389, 2000.

[5] G. Nickenig, A. T. Baumer, Y. Temur, D. Kebben, F. Jockenhovel, and M. Böhm, "Statin-sensitive dysregulated AT1 receptor function and density in hypercholesterolemic men," Circulation, vol. 100, no. 21, pp. 2131-2134, 1999.

[6] M. Horiuchi, T. X. Cui, Z. Li, J. M. Li, H. Nakagami, and M. Iwai, "Fluvastatin enhances the inhibitory effects of a selective angiotensin II type 1 receptor blocker, valsartan, on vascular neointimal formation," Circulation, vol. 107, no. 1, pp. 106-112, 2003.

[7] P. van der Harst, L. J. Wagenaar, H. Buikema et al., "Effect of intensive versus moderate lipid lowering on endothelial function and vascular responsiveness to angiotensin II in stable coronary artery disease," The American Journal of Cardiology, vol. 96, no. 10, pp. 1361-1364, 2005. 
[8] R. U. Pliquett, K. G. Cornish, J. D. Peuler, and I. H. Zucker, "Simvastatin normalizes autonomic neural control in experimental heart failure," Circulation, vol. 107, no. 19, pp. 24932498, 2003.

[9] S. Arita, N. Arita, and Y. Hikasa, "Effect of pravastatin on echocardiographic circulation parameters in dogs," The Journal of Veterinary Medical Science, vol. 76, no. 4, pp. 481-489, 2014.

[10] M. Borgarelli, P. Savarino, S. Crosara et al., "Survival characteristics and prognostic variables of dogs with mitral regurgitation attributable to myxomatous valve disease," Journal of Veterinary Internal Medicine, vol. 22, no. 1, pp. 120-128, 2008.

[11] W. Moonarmart, A. Boswood, V. Luis Fuentes, D. Brodbelt, K. Souttar, and J. Elliott, "N-terminal pro B-type natriuretic peptide and left ventricular diameter independently predict mortality in dogs with mitral valve disease," The Journal of Small Animal Practice, vol. 51, no. 2, pp. 84-96, 2010.

[12] A. Giaid and D. Saleh, "Reduced expression of endothelial nitric oxide synthase in the lungs of patients with pulmonary hypertension," The New England Journal of Medicine, vol. 333, no. 4, pp. 214-221, 1995.

[13] A. Giaid, M. Yanagisawa, D. Langleben et al., "Expression of endothelin-1 in the lungs of patients with pulmonary hypertension," The New England Journal of Medicine, vol. 328, no. 24, pp. 1732-1739, 1993.

[14] M. K. Jain and P. M. Ridker, "Anti-inflammatory effects of statins: clinical evidence and basic mechanisms," Nature Reviews Drug Discovery, vol. 4, no. 12, pp. 977-987, 2005. 